\title{
Evaluation of Aggregate and Silicone-oil Counts in Pre-filled Siliconized Syringes: An orthogonal study characterising the entire subvisible size range.
}

Maryam Shah ${ }^{\mathrm{a}, \#}$, Zahra Rattray ${ }^{\mathrm{a}, \mathrm{b}, \#, \text { Katie Day }}{ }^{\mathrm{c}}$, Shahid Uddin ${ }^{\mathrm{c}}$, Robin Curtis ${ }^{\mathrm{d}}$, Christopher F. van der Walle ${ }^{\mathrm{c}}$ and Alain Pluen ${ }^{\mathrm{a}^{*}}$

a , Division of Pharmacy and Optometry, School of Health Sciences, Faculty of Biology, Medicine and Health, University of Manchester, Manchester, UK

b, Present address: Department of Therapeutic Oncology, Yale School of Medicine, New Haven, USA

', MedImmune Ltd, Formulation Sciences, Granta Park, Cambridge, UK

d, School of Chemical Engineering and Analytical Sciences, University of Manchester, Manchester, UK

\#, authors contributed equally

*, corresponding author: Alain.Pluen@manchester.ac.uk 


\begin{abstract}
Characterisation of particulates in therapeutic monoclonal antibody (mAb) formulations is routinely extended to the sub-visible size-range (0.1-10 $\mu \mathrm{m})$. Additionally, with the increased use of pre-filled syringes (PFS), particle differentiation is required between proteinaceous and non-proteinaceous particles such as silicone-oil droplets. Here, three orthogonal techniques: Raster Image Correlation Spectroscopy (RICS), Resonance Mass Measurements (RMM) and Micro-Flow Imaging (MFI), were evaluated with respect to their sub-visible particle measurement and characterisation capabilities. Particle formation in mAb PFS solutions was evaluated with increasing polysorbate-20 (PS-20) concentrations. All three techniques provided complementary but distinct information on protein aggregate and silicone-oil droplet presence. PS-20 limited the generation of $\mathrm{mAb}$ aggregates during agitation, while increasing the number of silicone-oil droplets (PS-20 concentration dependant). MFI and RMM revealed PS-20 lead to the formation of larger micron-sized droplets, with RICS revealing an increase in smaller sub-micron droplets. Subtle differences in data sets complicate the apparent correlation between silicone-oil sloughing and mAb aggregates' generation. RICS (though the use of a specific dye) demonstrates an improved selectivity for $\mathrm{mAb}$ aggregates, a broader measurement size-range and smaller sample volume requirement. Thus, RICS is proposed to add value to the currently available particle measurement techniques and enable informed decisions during $\mathrm{mAb}$ formulation development.
\end{abstract}

Key words: particle, monoclonal antibody, protein aggregation, silicone-oil, primary packaging, raster image correlation spectroscopy, 


\section{Introduction}

There is an estimated production of 3.5 billion pre-filled syringe (PFS) units per year for therapeutic biopharmaceutical drug (e.g. monoclonal antibody (mAb)) administration, with a potential to grow to 6.7 billion units by 2020 (TMR, 2013)'(Rapra, 2015). The increase in PFS use is driven by factors such as the ease of use, advantages in safety, reductions in drug overfill and patient selfadministration; all of which reduce the incidence of hospitalisation and associated costs (Condino, 2005).

One of the challenges for the formulation scientist is to ensure the stability of the formulated $\mathrm{mAb}$ throughout the products lifetime, in the preferred presentation. Protein aggregation has been found to arise during and after fill-finish steps; which may develop from mechanical and/or agitation stress or from interaction with primary packaging components (Baldwin, 1988). Silicone-oil is a widelyutilised lubricant in PFS, facilitating ease of plunger movement in syringes and injection with hypodermic needles (Thirumangalathu et al., 2009); however, exposure to sloughed silicone-oil droplets has been suggested to adversely impact formulation stability (Gerhardt et al., 2014; Shi and Ladizhansky, 2012). Initial indication of adverse effects from silicone-oil was found in the 1980s following correlation of insulin particle formation with elevated blood glucose levels, in diabetics administered with the product (Baldwin, 1988). Later studies on agitation stress have shown the loss of soluble protein in PFS to be a particular problem during transportation (Gerhardt et al., 2014). Furthermore, agitation at higher speeds was correlated with an increase in monomer loss in reported shaking studies (Thirumangalathu et al., 2009). Subsequently, a number of silicone-oil related mechanisms underlying particulate formation have been proposed, exemplified by dispersed droplets acting as nucleation sites for protein aggregation (Majumdar et al., 2011); adsorption-destabilization of protein onto the silicone-oil/water interface (Thirumangalathu et al., 2009); and silicone-oil droplet surface charge neutralisation by adsorbed proteins resulting in agglomeration (Basu et al., 2013; Ludwig et al., 2010).

The size range of protein and silicone-oil particulates is generally wide (Table 1 presents the various size ranges and common terminologies used) (Ludwig et al., 2011; Philo, 2006; Philo, 2009; Weinbuch et al., 2013b). The United States Pharmacopeia (USP) chapter 'Particulate Matter in Injections' $<788>$ defines concentration limits for particles in parental solutions that are $\geq 10$ and 25 $\mu \mathrm{m}$ (Pharmacopeia, 2012b). USP chapter 'Subvisible Particulate Matter in Therapeutic Protein Injections' $<787>$ makes the recommendation to monitor particles $<10 \mu \mathrm{m}$, with a supporting chapter $<1787>$ giving guidance on the expanded techniques that can be used and size ranges (Pharmacopeia, 2012a). Based on the USP recommendations, the commercially available Micro-Flow Imaging (MFI) system, detecting particles from approximately $1 \mu \mathrm{m}$ to $400 \mu \mathrm{m}$ (Zolls et al., 2012)'(Sharma et al., 
2010b), is commonly used in the industry to assess sub-visible particulates alongside more established USP methods such as light obscuration (Pharmacopeia, 2012a, b). The potential immunogenic risk of smaller sub-visible aggregates $(0.1-10 \mu \mathrm{m})$ has been discussed by Carpenter et al (Carpenter et al., 2009) and Singh et al (Singh, 2013; Singh et al., 2010) and regulatory submissions therefore may include quantitative characterisation of micron-sized aggregates $(1-10 \mu \mathrm{m})$ and qualitative characterisation of sub-micron aggregates $(0.1-1 \mu \mathrm{m})$ in the early stages of development (Pharmacopeia, 2011; Weinbuch et al., 2013b). With the current particle detection technologies, an 'analytical gap' around $1 \mu \mathrm{m}$ still remains; consequently there is a drive for the development of new particle metrology tools (Gross et al., 2016). Furthermore, there is a high interest in developing technologies which are also capable of particle differentiation i.e. between protein and foreign matter, such as silicone-oil. In response to this predicament, in the last decade several new analytical technologies have been introduced in order to detect and characterise aggregates; offering the capability to extend the detectable size range of particles from $30 \mathrm{~nm}$ to $10 \mu \mathrm{m}$, through combining orthogonal technologies (Anacelia Ríos Quiroz 2015). For example, the recently developed Resonance Mass Measurement (RMM) system (Archimedes) has been utilised alongside MFI, as a particle metrology tool to bridge the analytical size 'gap' for particulates in the $0.5-5 \mu \mathrm{m}$ size range, and similar to MFI, discriminate between silicone-oil droplets and protein aggregates. However, the focus of the study was on large sub-micron and micron-sized particles through the utilisation of the RMM 'micro sensor', with a lower detection limit of $0.5 \mu \mathrm{m}$ (Pharmacopeia, 2011; Weinbuch et al., 2013b).

Table 1: Common terminology used for various protein aggregate size ranges (Carpenter et al., 2009; Narhi et al., 2012; Ríos Quiroz et al., 2015; Zolls et al., 2012).

\begin{tabular}{ll}
\hline Common terms & Size in Diameter \\
\hline Nano-metre aggregate, oligomer & $<100 \mathrm{~nm}$ \\
Sub-micron aggregates & $0.1-1 \mu \mathrm{m}$ \\
Smaller sub-visible aggregates & $0.1-10 \mu \mathrm{m}$ \\
Sub-visible particles, micron aggregates & $1-100 \mu \mathrm{m}$ \\
Visible particles & $>100 \mu \mathrm{m}$ \\
Analytical size gap & $0.5-5 \mu \mathrm{m}$ \\
\hline
\end{tabular}

Raster Image Correlation spectroscopy (RICS) is an image analysis tool, originally developed by Digman et al (Digman et al., 2005). We recently reported a comparison of particle size distributions in the gap region with the novel application of RICS, by extrinsic aggregate labelling, against Dynamic Light Scattering (DLS) and MFI, in simple mAb formulations (e.g. in the absence of silicone-oil and surfactant). RICS was demonstrated to measure a broad particle size range (i.e. $10 \mathrm{~nm}$ - $100 \mu \mathrm{m}$ ) for stressed mAb samples (i.e. thermal and freeze-thaw stress) (Hamrang et al., 2015); thereby providing scope for the application of RICS in more complex formulations. 
This manuscript reports the quantitative evaluation of protein and silicone-oil particulates formed in PFS solutions, both within and outside the analytical size gap range. We compare the complementary of RICS, detecting particles from $30 \mathrm{~nm}-10 \mu \mathrm{m}$, against RMM and MFI which are capable of particle sizing over the sub-micron $(\sim 0.1-\sim 5 \mu \mathrm{m}$, through the use of the nano and micro sensor) and micron (>1 $\mu \mathrm{m}$ ) sizes ranges, respectively. The PFS solutions, in the presence and absence of polysorbate-20 (PS-20), were subjected to agitation stress via end-over-end rotation, used to model stress during transportation (Gerhardt et al., 2014; V, 2011). There are numerous studies assessing the mechanisms of mAb aggregation (Li et al., 2011; Morris et al., 2009) and the effects of silicone-oil (Basu et al., 2013; Gerhardt et al., 2014; Jones et al., 2005; Weinbuch et al., 2013b) or polysorbate surfactants (Agarkhed et al., 2013; Khan et al., 2015) in influencing the aggregation process; such studies include novel methods to reduce in situ mAb aggregation in PFS (Depaz et al., 2014). However, the focus has been the larger sub-visible size range of particulates i.e. $>0.5 \mu \mathrm{m}$ (Felsovalyi et al.; Krayukhina et al., 2015; Teska et al.); due to the current lack of available technologies that are sensitive to the detection of smaller particles, whilst capable of differentiating between proteinaceous and foreign particulates (e.g. silicone-oil). Herein, the ability of RICS to characterise aggregates in solutions containing silicone-oil droplets via extrinsic fluorescent dyes is also evaluated: the selectivity of RICS (through the use of a specific dye) is compared with the efficiency of RMM and MFI (based on particle buoyancy and optical parameters for RMM and MFI, respectively) in particle differentiation. The assessment of size and concentration of particulates generated in siliconized PFS containing formulated $\mathrm{mAb}$ is reported utilising all three techniques. 


\section{Materials and Methods}

\subsection{Materials}

A bi-specific monoclonal antibody, herein termed 'COE-08', was kindly provided by Medimmune (Cambridge, UK). $1 \mathrm{~mL}$, long, sterile, ready to fill $\mathrm{BD}$ Hypak $^{\mathrm{TM}}$ glass siliconized syringes were purchased from Becton Dickinson and Company (New Jersey, US).

All buffer components including sucrose, L-histidine and PS-20 were of analytical grade or higher, purchased from Sigma Aldrich (Dorset, UK) and used without further purification.

SYPRO® Red and SYPRO® Orange dyes were obtained from Thermo Scientific (Leicestershire, UK) at a concentration of 5000× (in DMSO). All buffers and solutions were prepared with Millipore de-ionised water (18 M $\Omega . c m)$ and pre-filtered prior to stress experiments.

\subsection{Methods}

\subsubsection{Sample Preparation}

All solutions were prepared in a pH 6 buffer composed of $25 \mathrm{mM}$ histidine and $235 \mathrm{mM}$ sucrose. COE-08 solutions were prepared at a final concentration of $10 \mathrm{mg} / \mathrm{mL}$ in the presence of $0,0.02$ and $0.05 \% \mathrm{w} / v$ PS-20 and placed in syringes. Control syringes were filled with buffer containing the same PS-20 concentrations i.e. in the absence of mAb. Solutions were placed in the syringes ensuring a consistently sized air bubble, of a height of approximately $1 \mathrm{~mm}$. Multiple syringes were used per condition to ensure sufficient sample volume for all three instruments. Syringes were placed on an end-over-end rotator at ambient temperature $\left(21^{\circ} \mathrm{C}\right)$ in a thermostatically-controlled environment for a 24 hour agitation period at $20 \mathrm{rpm}$. In parallel, non-agitated samples (of the same described solutions) were stored in an open rack on the benchtop.

\subsubsection{Analysis of Particulates with Confocal Microscopy (RICS)}

SYPRO® Red and SYPRO® Orange (Thermo Scientific, Leicestershire, UK), used to label protein aggregates and silicone-oil droplets, respectively, were added to samples (post-experiment) 15 minutes prior to visualisation with confocal microscopy at a final working concentration of $2.5 \times$.

A Zeiss 510 Confocor 2 (Zeiss, Jena, Germany) confocal microscope equipped with a c-Apochromat 40×/1.2NA water-immersion objective was utilised for image acquisition. For SYPRO® Red solutions, imaging was carried out by exciting the dye with a Helium-Neon laser at $543 \mathrm{~nm}$ and the emitted fluorescence collected above $585 \mathrm{~nm}$ (LP585 filter set). Excitation of SYPRO Orange ${ }^{\circledR}$ was carried out at $488 \mathrm{~nm}$ (Argon laser) and the emitted fluorescence collected with a 560-615 nm bandpass filter. Confocal image time series of 1,024 × 1,024 pixel resolution were captured over 100 frames with a corresponding pixel dwell time of 6.4 microseconds. In-house RICS software (ManICS) was applied to analysis of images acquired using confocal microscopy. A full description of the RICS 
algorithm has been described elsewhere (Digman et al., 2005; Hamrang et al., 2012). The aforementioned image time series were sub-divided into $32 \times 32$ pixels region of interest (ROI) and the diffusion coefficients (D) within each ROI was generated (Figure 1a). All fits possessing a $\mathrm{R}^{2}$ below 0.7 were discarded from the fit data prior to generation of particle size distributions.

RICS-derived diffusion coefficients were subsequently converted to particle diameter using the StokeEinstein equation (following determination of solvent viscosity):

$D=k T / 3 \pi \eta a$

Equation 1

Where $D$ refers to the diffusion coefficient, $k$ refers to the Boltzmann constant, $T$ the temperature at which the measurements were performed, $\eta$ solvent viscosity and $a$ the hydrodynamic diameter.

\subsubsection{Resonant Mass Measurement (RMM)}

Particle size analysis using RMM is based on frequency shifts that are proportional to particle buoyant mass, and depend on the sensitivity of a resonator (Weinbuch et al., 2013a). An Archimedes system (Malvern, UK) was utilised for RMM of positively- (silicone-oil droplets) and negatively- buoyant (protein aggregates) particles. Both the nano and micro sensor were utilised for all solutions. The limit of detection (LOD) was set at $0.01 \mathrm{~Hz}$ (corresponding to $0.07 \mu \mathrm{m}$ for protein particles and $0.17 \mu \mathrm{m}$ for silicone-oil particles) and $0.03 \mathrm{~Hz}$ (corresponding to $0.33 \mu \mathrm{m}$ for protein particles and $0.68 \mu \mathrm{m}$ for silicone-oil particles) for the nano and micro sensors, respectively. System set-up and cleaning procedures are described by the manufacturer and elsewhere (Depaz et al., 2014; Weinbuch et al., 2013b).

\subsubsection{Micro-flow Imaging (MFI) Analysis}

MFI analysis was performed using a Protein Simple MFI 5000 series (Protein Simple, California, USA). Millipore filtered pure water and particle-free buffer $(5 \mathrm{~mL})$ was purged through the system to remove residual particles prior to measurements and reduce the baseline prior to data acquisition for each sample. Subsequently, the sample was introduced at a flow rate of $0.5 \mathrm{~mL} / \mathrm{min}$, the illumination optimized and $0.5 \mathrm{~mL}$ of sample analysed at a corresponding flow rate of $0.1 \mathrm{~mL} / \mathrm{min}$. Bright-field images, morphometric (i.e. equivalent circle diameter (ECD) and aspect ratio) and particle data obtained from the analysis of agitated and non-agitated samples were subjected to analysis of particle counts, morphology and size distribution.

A customised filter was adapted from previous studies (Strehl et al., 2012; Weinbuch et al., 2013a) and applied to differentiate between silicone-oil and proteinaceous particles using Origin 2016 (OriginLab Corporation, Northampton, MA, USA). MFI data obtained from solutions containing silicone-oil only or COE-08 aggregates only were utilised to create a customised discriminant analysis filter based on four MFI parameters: aspect ratio, intensity mean, intensity minimum and intensity 
standard deviation ( $c f$. Sharma et al. (Sharma et al., 2010c)). Discriminant analysis uses known sample to build a model that can aid data stratification through establishing particle identity (i.e. COE08 or silicone-oil). The analysis was applied to each MFI-generated dataset. To set the mAb standard during development of the customised filter, an aliquot of COE-08 in buffer was subjected to agitation via end-over-end rotation (for 24 hours) in de-siliconized syringes.

\subsubsection{Statistical Analysis}

Unless otherwise stated, a non-parametric one-way ANOVA was performed to assess the influence of stress type on resultant size distribution/particle counts. A calculated probability (i.e. p-value) equal or less than 0.05 was considered to be statistically significant. 


\section{Results}

The formation of protein aggregates and silicone-oil droplets in PFS, in the presence and absence of agitation stress, was evaluated as a function of PS-20 concentration $(0,0.02$ and $0.05 \% \mathrm{w} / \mathrm{v})$. MFI, RMM and RICS were utilized, as described in the methods, to selectively evaluate protein aggregate formation and silicone-oil sloughing, over the broadest size range. For each technique, the particle counts were separated, where applicable, into size ranges of (i) $<0.07 \mu \mathrm{m}$ (RICS only), (ii) 0.07-0.5 $\mu \mathrm{m}$ (RICS and RMM), (iii) 0.5-5 $\mu \mathrm{m}$ (RICS, RMM and MFI; with MFI only detecting particles > 1 $\mu \mathrm{m}$ ), and (iv) > $5 \mu \mathrm{m}$ (RICS, RMM and MFI). The size ranges were chosen with respect to the RMM nano sensor analytical range of 0.07-0.5 $\mu \mathrm{m}$.

\subsection{Fluorescent dye selection for proteinaceous aggregates and silicone-oil droplets for RICS analysis}

Since confocal microscopy and RICS analysis rely on fluorophores, RICS may distinguish between particles originating from different materials (in this case, protein $v s$ silicone-oil) provided a dye with relevant physicochemical properties is selected. SYPRO® Red was previously used to label protein aggregates in simple formulations (Hamrang et al., 2015). In this study, SYPRO® Red was used to label protein aggregates in more complex formulations (i.e. in the presence of silicone-oil and / or surfactant micelles).

Micrographs (Figure 1b) suggested no apparent labelling of silicone-oil and / or PS-20 micelles by SYPRO® Red that would interfere with the data obtained from labelled protein aggregates. Moreover, following RICS analysis, the images acquired of buffer-only PFS solutions (i.e. in absence of mAb), did not generate any conclusive data as an insufficient correlation (with $\mathrm{R}^{2}<0.7$ ) was obtained. This result indicated the significantly higher affinity of SYPRO® Red for proteinaceous aggregates compared to silicone-oil droplets and/or PS-20 micelles.

A second dye was required for labelling silicone-oil droplets. As an initial search did not reveal a fluorophore capable of selectively labelling silicone-oil droplets (but not proteinaceous aggregates), SYPRO ${ }^{\circledR}$ Orange was assessed in labelling silicone-oil droplets in buffer-only PFS solutions i.e. in the absence of mAb (COE-08) (Nashine et al., 2013; Vedadi et al., 2010). Micrographs (Figure 1b) illustrated labelling of silicone-oil droplets by SYPRO® Orange. Following RICS analysis of the acquired buffer-only PFS solution images (with SYPRO® Orange), sufficient correlations (with $\mathrm{R}^{2}>$ 0.7) were obtained. Thus, RICS analysis (with SYPRO® Orange) of silicone-oil droplets was assessed in non-mAb solutions only.

To clarify, for RICS analysis, SYPRO® Red was utilised to label protein aggregates in mAb PFS solutions, and SYPRO ${ }^{\circledR}$ Orange for labelling silicone-oil droplets in mAb-free PFS solutions.

\section{Figure 1}




\subsection{Assessment of mAb Aggregation in Siliconized PFS}

Protein particle counts (particle counts per $\mathrm{mL}$ ) by each technique (RICS, RMM and MFI) are presented in Figure 2. A broad range of COE-08 aggregate sizes is illustrated in the non-agitated (Figure 2, left) and agitated (Figure 2, right) PFS solutions consistent with our previous work (Hamrang et al., 2015). The size distributions of the protein particles, clearly showing the outliers, can be seen in the SI (Figure S1).

When assessing the particle profiles of the three techniques (Figure 2), complementary is observed in relation to (i) higher aggregate counts in the absence of PS-20 following agitation and (ii) the greater presence of smaller particles: significantly higher $(\mathrm{p}<0.05)$ absolute aggregate counts were measured in agitated PFS solutions in the absence of PS-20, by all three techniques. An overall assessment of the separated particle size ranges indicates that the larger the particle size, the smaller the observed particle count will be (a typical trend already found in aggregate solutions) (Ripple and Narhi, 2015; Singh et al., 2010). In relation to this assessment, the two main observations were, (i) significantly lower absolute particle concentrations (particles per $\mathrm{mL}$ ) detected by MFI in comparison to RMM, for all PFS solutions (approximately three orders of magnitude) and (ii) the higher absolute aggregate counts in the $0 \% w / v$ PS-20 agitated samples were due to the significantly higher particle counts $(\mathrm{p}<$ $0.05)$ in the $0.5-5 \mu \mathrm{m}$ size range by MFI and significantly higher particle counts $(\mathrm{p}<0.01)$ in the $0.07-0.5 \mu \mathrm{m}$ size range by RMM and RICS.

Additionally, when assessing the particle size ranges across the three techniques, a pattern is observed when considering each technique's analytical capability, the sampled volume, and the trend of low incidence of larger particles. MFI utilises the largest sampled volume and detected particles $>5 \mu \mathrm{m}$ in all PFS solutions. By RICS and RMM, only the 0\% $w / v$ PS-20 agitated PFS solutions contained particles larger than $5 \mu \mathrm{m}$ in diameter. The same sample when analysed by MFI contained the highest particle concentration in the $>5 \mu \mathrm{m}$ size range. Similarly, by RICS only the agitated solutions contained particles in the 0.5-5 $\mu \mathrm{m}$ size range; and the same samples contained higher particle counts by RMM and MFI, in comparison to the non-agitated solutions (Figure 2).

\section{Figure 2}




\subsection{Characterisation of Dispersed Silicone-oil in PFS}

\subsubsection{Silicone-oil Droplets in PFS Containing Buffer Only (no mAb)}

Dispersed silicone-oil droplets in buffer-filled non-agitated and agitated PFS solutions, in the presence of $0,0.02$ and $0.05 \% w / v$ PS-20, were characterized by RMM, RICS and MFI as described in the methods. Silicone-oil droplet counts are presented in Figure 3 and the size distributions can be seen in the SI (Figure S2).

It is observed that the presence of PS-20 resulted in significantly higher total droplet counts $(\mathrm{p}<$ 0.05), for non-agitated and agitated PFS solutions (Figure 3). This is an interesting outcome as the PS20 solutions had the lowest protein aggregate counts, as seen in Figure 2. The results illustrate that PS-20 had a more dominant effect than agitation in the sloughing of silicone-oil in PFS.

Similarly to the mAb profiles, higher concentrations of smaller sized oil droplets are detected by all three techniques (Figure 3), for all solutions. The smaller size ranges for RMM (i.e. 0.07-0.5 $\mu \mathrm{m}$ ) and RICS $(<0.07$ and $0.07-0.5 \mu \mathrm{m})$ detected significantly higher oil droplet counts $(\mathrm{p}<0.05)$ in the presence of PS-20 (non-agitated and agitated solutions). Larger silicone-oil droplets i.e. > $5 \mu \mathrm{m}$, detected by MFI, were also greater in presence in PS-20 agitated samples.

As with the mAb data in Figure 2, the differences in sampled volumes across the techniques and the low incidence of larger particles may explain the results in the overlapping size ranges. For example, RMM only measured particles larger than $5 \mu \mathrm{m}$ in the $0.05 \% w / v$ PS-20 PFS solutions (non-agitated and agitated). RICS detected silicone-oil particles in the $0.5-5 \mu \mathrm{m}$ size range in the presence of PS-20 $(0.02$ and $0.05 \% \mathrm{w} / \mathrm{v})$ or following agitation, and only detected silicone-oil particles larger than $5 \mu \mathrm{m}$ in the $0.05 \% w / v$ PS-20 agitated solutions (Figure 3).

\section{Figure 3}




\subsubsection{RMM and MFI Characterisation of Silicone-oil Droplets in mAb PFS Samples}

Silicone-oil droplet counts in the mAb PFS solutions were determined by RMM and MFI (distinguishing between proteinaceous particles and silicone oil droplets as described in the methods) and are presented in Figure 4. The silicone-oil size distributions can be seen in the SI (Figure S3).

Both RMM and MFI showed a greater concentration of larger silicone-oil droplets in the presence of PS-20 following agitation (PS-20 concentration dependant): similar to the buffer-filled PFS data (Figure 3), the only solutions containing particles larger than $5 \mu \mathrm{m}$ by RMM were in the presence of PS-20. Following agitation, MFI detected a higher concentration of particles larger than $5 \mu \mathrm{m}$ in the PS-20 samples. Conversely, unlike RMM, MFI data showed differences in silicone-oil droplet counts between the mAb PFS (Figure 4) and buffer-only PFS solutions (Figure 3): significantly higher total droplet counts were observed in the $0 \% w / v$ PS-20 agitated mAb PFS solutions (Figure 4). Particle size separation showed this was due to the significantly higher particle counts in the 0.5-5 $\mu \mathrm{m}$ size range $(\mathrm{p}<0.01)$.

It is important to note that differentiation of protein and silicone-oil particles with MFI proved problematic; even with the use of the discriminant analysis described in the methods. This is a problem observed in previous papers due to optical similarities between protein and silicone-oil particles with a diameter less than $4 \mu \mathrm{m}$ (Zölls et al., 2013; Zolls et al., 2012). Table 2 presents the (apparent) protein concentrations in buffer-only (i.e. mAb-free) PFS solutions determined by MFI, following discriminant analysis. The apparent protein concentrations determined for the non-agitated and agitated PFS solutions were above the background count limit of the MFI system i.e. the threshold for a clean run of water (determined as 200 particles per $\mathrm{mL}$ ). Thus, particle differentiation issues between protein and silicone-oil following discriminant-analysis are indicated. This result is exacerbated for particle numbers in the lower size range, i.e. $0.5-5 \mu \mathrm{m}$; as the concentration for particles $>5 \mu \mathrm{m}$ is less than the background limit, unlike the $0.5-5 \mu \mathrm{m}$ size range. Thus it is possible that the (apparent) higher silicone-oil particles per $\mathrm{mL}$ in the 0\% (w/v) PS-20 (mAb PFS) agitated solutions (Figure 4) could be a result of particle differentiation issues between protein and silicone-oil in the lower MFI size-range in this study (i.e. 0.5-5 $\mu \mathrm{m}$ ).

Table 2: Apparent protein concentrations in buffer-filled PFS solutions determined by MFI (following discriminant analysis). Values represent averages with std. dev. for $n=3$.

\begin{tabular}{llll}
\hline Solution & Total $(1-10 \mu \mathrm{m})$ & $1-5 \mu \mathrm{m}$ & $>5 \mu \mathrm{m}$ \\
\hline Buffer in PFS Non-Agitated & $1796 \pm 393$ & $1711 \pm 380$ & $85 \pm 27$ \\
Buffer in PFS Agitated & $4133 \pm 313$ & $4004 \pm 370$ & $129 \pm 57$ \\
\hline
\end{tabular}

\section{Figure 4}




\section{Discussion}

\subsection{Considerations Regarding the Different Techniques}

The information gained from the various commercially-available particle metrology technologies has recently been subjected to considerable discussion, as highlighted by Ripple and Dimitrova (Ripple and Dimitrova, 2012) and Quiroz et al (Ríos Quiroz et al., 2015). Common to all techniques extrapolating data obtained from small sample volumes or in dilute samples, is the uncertainty in particle-size data approaching the technique detection limit. The substantial differences in sampled volumes across the three techniques in the present study: RICS $\left(\sim 2 \times 10^{-9} \mathrm{~mL}\right)<\mathrm{RMM}\left(\sim 4 \times 10^{-6} \mathrm{~mL}\right.$ and $\sim 1 \times 10^{-4} \mathrm{~mL}$ by the nano and micro sensors, respectively $)<\operatorname{MFI}\left(3 \times 10^{-1} \mathrm{~mL}\right)$, may explain the differences observed in the data in the overlapping size ranges between the techniques. Low sampled volumes reduce the likelihood of detecting larger particles present in low concentrations (Anacelia Ríos Quiroz 2015). On the other hand, a significant sample volume is required to generate statistically significant particle counts per dose unit. Consequently, the argument for poor precision of new techniques in favour of light obscuration is debatable since all techniques are known to suffer from caveats. For example, in the case of HIAC and MFI, both of which are optical-based particle counting techniques, the techniques are influenced by the refractive index difference between protein particles and the formulation. As highlighted by Ripple and $\mathrm{Hu}$ (Ripple and Hu, 2015) and Zölls et al (Zölls et al., 2013), the change in refractive index at higher concentrations has led to the underestimation of particle concentrations. Hence, although novel and emerging techniques may not be appropriate for quality control in their current state, they are capable of monitoring the early stages of aggregation, require minimal sample volume and are therefore directly relevant to early stages of formulation development. Thus the main focus of the present work was to assess the presence of particulates that may not be easily detected by light obscuration or MFI i.e. in the submicron size range and smaller. This manuscript compares the particle trends / concentrations across MFI, RMM (micro and nano sensor) and RICS.

MFI has received much attention in the analysis of large protein particles (i.e. $>1 \mu \mathrm{m}$ ) (Sharma et al., 2010a; Sharma et al., 2010c; Wuchner et al., 2010) as the volume and the size-range matches regulations; in regards to their morphology, and recently in differentiating between protein and silicone-oil particles using customised filters (Strehl et al., 2012; Weinbuch et al., 2013b). The discriminant analysis used in this study is based on certain particle parameters relating to apparent optical properties and circularity (aspect ratio, intensity mean, intensity minimum and intensity standard deviation), devised by Weinbuch et al (Weinbuch et al., 2013b). However, due to some optical similarities between $\mathrm{mAb}$ and silicone-oil, the reliability of this analysis has previously been questioned for particles < $4 \mu \mathrm{m}$ (Weinbuch et al., 2013b; Zölls et al., 2013; Zolls et al., 2012). Supporting previous literature, this study observed a significant apparent presence of COE-08 particles above the background count limit in mAb-free PFS solutions (Table 2). The misclassification 
error with MFI may offer some explanations for the possible effects observed in the $0.5-5 \mu \mathrm{m}$ size range in this study i.e. accounting for the higher oil droplet concentration in the $0 \% \mathrm{w} / \mathrm{v}$ PS-20 agitated solutions in Figure 4, which is inconsistent with other data-sets. Thereby, for mixed solutions (i.e. protein and silicone-oil), it is recommended to utilise MFI alongside another method possessing an overlapping size range (covering 1-5 $\mu \mathrm{m}$ ) and also capable of particle differentiation i.e. RMM (Weinbuch et al., 2013b; Zolls et al., 2012).

Overall, results from the current study are consistent with previous reports showing complementarity between RMM and MFI (Weinbuch et al., 2013b) (Figure 2 for protein aggregates, and Figures 3-4 for silicone-oil droplets). This is true when accounting for the strong dependence between number of particles and their sizes (Ríos Quiroz et al., 2015; Ripple and Narhi, 2015), wherin a significantly greater aggregate count in the lower sub-visible size range was measured following and prior to agitation in PFS. Through the use of the RMM nano sensor and RICS, the smaller-sized aggregate population was analysed, detecting particles $<0.5 \mu \mathrm{m}$ in diameter. There are limited published reports on the use of RMM (Panchal et al., 2014; Weinbuch et al., 2013b; Zölls et al., 2013), and to our knowledge, this is the first study reporting the use of both RMM sensors on the same solutions. A careful observation of size ranges detected by RMM micro and nano sensors (SI, Figure S1) revealed uncertainties regarding the use of both detectors. While the size ranges of the nano and micro sensors are intended to overlap, an overlap of particle sizes is not always observed, raising questions about the likelihood of detecting poorly-populated larger particles in the small sampled volume. The same observation was found in the silicone-oil data acquired by RMM (Figure S3).

It is noteworthy that RMM exploits the differences in density to distinguish between particles but requires the use of both the nano and micro sensors to cover a broad size range, which increases measurement time and sample consumption. Furthermore, RMM has particle concentration limits, accruing errors for samples with low particle counts (Weinbuch et al., 2013b), but also for samples with particle counts $>2 \times 10^{6}$ particles $/ \mathrm{mL}$ where there is a risk of high coincidence and dilution is required (Amin et al., 2014; Panchal et al., 2014). Concentration limits appear to be a downfall for many technologies characterising in the lower size range. For example, Nano-Particle Tracking Analysis (NTA), which also has the ability of particle differentiation through using fluorescence, has similar concentration limits to RMM. The combined effect of adsorption (from contact with glass and stainless steel during measurement) and sheer (during injection) has also been reported to create aggregates (Filipe et al., 2010; Funke et al., 2016).

With regard to RICS, the main challenge was the selection of an appropriate dye. In this study, the selectivity of SYPRO ${ }^{\circledR}$ Red for protein aggregates was demonstrated, with SYPRO® Orange labelling both protein aggregates (Goldberg et al., 2011; He et al., 2010; Nashine et al., 2013) and silicone-oil droplets (Figure 1). When using RICS, fluorescent dye selection should be considered on a case-by-case basis. However, following the selection of the fluorophore, no change of detector was 
required and the trends for particle size and concentration observed with RMM were consistent with those observed by RICS.

It should be mentioned that comparison of particle concentrations from MFI, RMM or RICS data needs consideration due to fundamental differences in measurement between these techniques. MFI and RMM both use flow that can potentially bias the movement of particulates such that particles are counted within a certain sampled volume. With the present settings, RICS detects a number of particles in the focal volume of the objective, but particles moving by Brownian motion may cross this focal volume more than once. Indeed, Figures 2 and 3 suggest higher particle counts than those determined by RMM and MFI. Thus, in the current setting, direct comparison of particle counts between RMM, MFI vs RICS cannot be made. It is foreseen that microfluidics may be of use with RICS; Rossow et al. have demonstrated the use of RICS in the presence of flow (Rossow et al., 2009).

It is important to consider the uncertainties carried by different technologies, especially when comparing the acquired data-sets across multiple techniques. Nevertheless, considering all of the above points, the trends across the three techniques i.e. the effect of agitation and the presence of PS20 on aggregation formation $v s$ the dispersion of silicone-oil droplets in the PFS solutions, are the same.

\subsection{Agitation in Siliconized PFS Increases Aggregation Formation}

All samples containing 0\% $w / v$ PS-20 contained a significantly larger aggregate count following agitation, which is consistent with the widely known effect that agitation has on protein solutions sheared at the air-water interface (Khan et al., 2015; Treuheit et al., 2002). Due to the increased use of PFS in fill-finish manufacturing, it is important to understand the impact of siliconized syringes on the stability of formulated $\mathrm{mAb}$ during storage and transport in the presence or absence of agitation. Previous literature indicate that the presence of silicone-oil can result in aggregation increase following agitation; the effect being silicone-oil concentration dependant (Jones et al., 2005). Other studies have indicated that silicone-oil itself i.e. in the absence of an additional stress such as agitation, does not impact aggregation formation (Gerhardt et al., 2014; Thirumangalathu et al., 2009). In this study, silicone-oil droplets and aggregated protein were detected in all mAb-filled (COE-08) PFS solutions (Figure 2 for aggregates and Figure 4 for silicone-oil). As the PS-20 solutions contained the lowest aggregate counts (Figure 2) whilst containing the highest silicone-oil droplet counts (Figures 3-4), no correlation between protein aggregation and silicone-oil droplet presence was observed. Based on this, and previous literature, it may be that the effect of silicone-oil on mAb stability is a case by case basis.

Considering the surface-active properties of PS-20, the observed increase of silicone-oil droplets generated in PS-20 samples (Figure 3) was consistent with previous reports (Felsovalyi et al., 2012). This observation needs to be tempered against the imaging method used for RICS which relies on 
inverted microscopy of a small sample volume within a narrowly-defined plane of focus across which droplets move by Brownian motion and by virtue of their density relative to the bulk. Here, we have assumed that PS-20 stabilised silicone-oil droplets in solution (Ludwig et al., 2010), and silicone-oil droplets in the absence of PS-20 have the same density and are therefore positively buoyant in aqueous solution. Nevertheless, silicone-oil extracted from the surface by PS-20 appears to increase the number of droplets in the micrometre region ('outliers' observed in Figure S2).

\subsection{PS-20 limits the Formation of Aggregates in Siliconized PFS following Agitation}

Agitated PFS solutions in the absence of PS-20 generated the highest protein aggregate counts following agitation (Figure 2). More so, the presence of PS-20 reduced the protein particle counts to their respective baselines, i.e. the counts in non-agitated solutions for the same PS-20 concentration. RICS and RMM showed that PS-20 significantly reduced small sub-visible aggregates, while MFI showed that PS-20 limited the development of larger aggregates (Figure 2). A number of studies have attempted to explain the protective mechanisms of polysorbates in preventing aggregation (see Khan et al (Khan et al., 2015)). The predominant mechanism is assumed to be adsorption competition between the surfactant and the protein at the air/liquid (or glass/liquid) interface. As a result, adsorption-denaturation of the protein at these interfaces is attenuated (Khan et al., 2015; Lee et al., 2011; Thirumangalathu et al., 2009). A further suggestion is that surfactant molecules may form micelles (at concentrations above the critical micelle concentration) that shield exposed protein hydrophobic surfaces, assuming partial or complete denaturation, and attenuate protein-protein interactions (Bam et al., 1998; Mahler et al., 2005).

Protein destabilising effects in increasing polysorbate concentrations have been observed in other studies (Agarkhed et al., 2013; Amand Bhaskar, 2014). In this study, no significant differences in aggregate formation were observed between the two PS-20 concentrations (Figure 2), although a higher concentration of silicone-oil droplets is observed in the $0.05 \% w / v$ concentration (Figures 3-4). Nevertheless, determining the optimal polysorbate concentration for a specific $\mathrm{mAb}$ formulation must be accounted for during formulation development.

\section{Conclusions}

All three techniques demonstrated that the presence of PS-20 in mAb solutions contributes to a significant reduction in proteinaceous aggregates following agitation, consistent with the surfactant activity of PS-20. Comparison of the data sets imply that there is no interplay between the sloughing of silicone-oil droplets in PFS and the exacerbation of protein aggregate formation in the sub-visible size range. While advanced particle characterisation technologies are available to the formulation scientist, it is still the case that this is a challenging area and emerging methods, while welcomed, may not have yet achieved the expected capability of bridging the current sizing 'gap' for sub-visible particles. Nevertheless, our data show that they provide complementary information and support 
methods such as MFI. This study demonstrates that RICS analysis may expand the scope for subvisible particle sizing/characterisation and is an orthogonal technique to RMM. Since confocal microscopy is well established, RICS offers the potential for widespread application in laboratories where specialist equipment may not be available.

\section{Acknowledgements}

MS was supported by a Biotechnology and Biological Sciences Research Council (BBSRC) 'BRIC' studentship with MedImmune Ltd. 


\section{References}

Agarkhed, M., O’Dell, C., Hsieh, M.-C., Zhang, J., Goldstein, J., Srivastava, A., 2013. Effect of Polysorbate 80 Concentration on Thermal and Photostability of a Monoclonal Antibody. AAPS PharmSciTech 14, 1-9.

Amand Bhaskar, K.M., Manish Kumar, 2014. Effect of Polysorbate-80 Concentration on GCSF Formulation Using Liquid Chromatography. International Journal of Pharmacy and Pharmaceutical Sciences 6, 299-302.

Amin, S., Barnett, G.V., Pathak, J.A., Roberts, C.J., Sarangapani, P.S., 2014. Protein aggregation, particle formation, characterization \&amp; rheology. Current Opinion in Colloid \& Interface Science 19, 438-449.

Anacelia Ríos Quiroz , J.L., Thierry Da Cunha , Adeline Boillon , Michael Adler, Christof Finkler, Joerg Huwyler, Roland Schmidt, Hanns-Christian Mahler, Atanas V. Koulov, 2015. Factors Governing the Precision of Subvisible Particle Measurement Methods - A Case Study with a Low-Concentration Therapeutic Protein Product in a Prefilled Syringe Pharm Res 33, 450-461.

Baldwin, R.N., 1988. Contamination of Insulin by Silicone Oil: a Potential Hazard of Plastic Insulin Syringes. Diabetic Medicine 5, 789-790.

Bam, N.B., Cleland, J.L., Yang, J., Manning, M.C., Carpenter, J.F., Kelley, R.F., Randolph, T.W., 1998. Tween protects recombinant human growth hormone against agitation-induced damage via hydrophobic interactions. Journal of Pharmaceutical Sciences 87, 1554-1559.

Basu, P., Krishnan, S., Thirumangalathu, R., Randolph, T.W., Carpenter, J.F., 2013. IgG1 aggregation and particle formation induced by silicone-water interfaces on siliconized borosilicate glass beads: A model for siliconized primary containers. Journal of Pharmaceutical Sciences 102, 852-865.

Carpenter, J.F., Randolph, T.W., Jiskoot, W., Crommelin, D.J.A., Russell Middaugh, C., Winter, G., Fan, Y.-X., Kirshner, S., Verthelyi, D., Kozlowski, S., Clouse, K.A., Swann, P.G., Rosenberg, A., Cherney, B., 2009. Overlooking Subvisible Particles in Therapeutic Protein Products: Gaps That May Compromise Product Quality. Journal of Pharmaceutical Sciences 98, 1201-1205.

Condino, A.A.F., Sara; Hoffenberg, Edward J., 2005. A Home Infliximab Infusion Program. Journal of Pediatric Gastroenterology and Nutrition 40, 67-69.

Depaz, R.A., Chevolleau, T., Jouffray, S., Narwal, R., Dimitrova, M.N., 2014. Cross-Linked Silicone Coating: A Novel Prefilled Syringe Technology That Reduces Subvisible Particles and Maintains Compatibility with Biologics. Journal of Pharmaceutical Sciences 103, 13841393.

Digman, M.A., Brown, C.M., Sengupta, P., Wiseman, P.W., Horwitz, A.R., Gratton, E., 2005. Measuring fast dynamics in solutions and cells with a laser scanning microscope. Biophysical Journal 89, 1317-1327.

Felsovalyi, F., Janvier, S., Jouffray, S., Soukiassian, H., Mangiagalli, P., 2012. Silicone-OilBased Subvisible Particles: Their Detection, Interactions, and Regulation in Prefilled 
Container Closure Systems for Biopharmaceuticals. Journal of Pharmaceutical Sciences 101, 4569-4583.

Filipe, V., Hawe, A., Jiskoot, W., 2010. Critical Evaluation of Nanoparticle Tracking Analysis (NTA) by NanoSight for the Measurement of Nanoparticles and Protein Aggregates. Pharm Res 27, 796-810.

Funke, S., Matilainen, J., Nalenz, H., Bechtold-Peters, K., Mahler, H.-C., Friess, W., 2016. Silicone Migration From Baked-on Silicone Layers. Particle Characterization in Placebo and Protein Solutions. Journal of Pharmaceutical Sciences 105, 3520-3531.

Gerhardt, A., McGraw, N.R., Schwartz, D.K., Bee, J.S., Carpenter, J.F., Randolph, T.W., 2014. Protein Aggregation and Particle Formation in Prefilled Glass Syringes. Journal of Pharmaceutical Sciences 103, 1601-1612.

Goldberg, D.S., Bishop, S.M., Shah, A.U., Sathish, H.A., 2011. Formulation development of therapeutic monoclonal antibodies using high-throughput fluorescence and static light scattering techniques: Role of conformational and colloidal stability. Journal of Pharmaceutical Sciences 100, 1306-1315.

Gross, J., Sayle, S., Karow, A.R., Bakowsky, U., Garidel, P., 2016. Nanoparticle tracking analysis of particle size and concentration detection in suspensions of polymer and protein samples: Influence of experimental and data evaluation parameters. European Journal of Pharmaceutics and Biopharmaceutics 104, 30-41.

Hamrang, Z., Hussain, M., Tingey, K., Tracka, M., Casas-Finet, J.R., Uddin, S., van der Walle, C.F., Pluen, A., 2015. Characterisation of Stress-Induced Aggregate Size Distributions and Morphological Changes of a Bi-Specific Antibody Using Orthogonal Techniques. Journal of Pharmaceutical Sciences 104, 2473-2481.

Hamrang, Z., Pluen, A., Zindy, E., Clarke, D., 2012. Raster image correlation spectroscopy as a novel tool for the quantitative assessment of protein diffusional behaviour in solution. Journal of Pharmaceutical Sciences 101, 2082-2093.

He, F., Phan, D.H., Hogan, S., Bailey, R., Becker, G.W., Narhi, L.O., Razinkov, V.I., 2010. Detection of IgG aggregation by a high throughput method based on extrinsic fluorescence. Journal of Pharmaceutical Sciences 99, 2598-2608.

Jones, L.S., Kaufmann, A., Middaugh, C.R., 2005. Silicone oil induced aggregation of proteins. Journal of Pharmaceutical Sciences 94, 918-927.

Khan, T.A., Mahler, H.-C., Kishore, R.S.K., 2015. Key interactions of surfactants in therapeutic protein formulations: A review. European Journal of Pharmaceutics and Biopharmaceutics.

Krayukhina, E., Tsumoto, K., Uchiyama, S., Fukui, K., 2015. Effects of Syringe Material and Silicone Oil Lubrication on the Stability of Pharmaceutical Proteins. Journal of Pharmaceutical Sciences 104, 527-535.

Lee, H.J., McAuley, A., Schilke, K.F., McGuire, J., 2011. Molecular origins of surfactantmediated stabilization of protein drugs. Advanced Drug Delivery Reviews 63, 1160-1171.

Li, Y., Mach, H., Blue, J.T., 2011. High throughput formulation screening for global aggregation behaviors of three monoclonal antibodies. Journal of Pharmaceutical Sciences 100, 2120-2135. 
Ludwig, D.B., Carpenter, J.F., Hamel, J.B., Randolph, T.W., 2010. Protein adsorption and excipient effects on kinetic stability of silicone oil emulsions. Journal of Pharmaceutical Sciences 99, 1721-1733.

Ludwig, D.B., Trotter, J.T., Gabrielson, J.P., Carpenter, J.F., Randolph, T.W., 2011. Flow cytometry: A promising technique for the study of silicone oil-induced particulate formation in protein formulations. Analytical Biochemistry 410, 191-199.

Mahler, H.-C., Müller, R., Frie $\beta$, W., Delille, A., Matheus, S., 2005. Induction and analysis of aggregates in a liquid IgG1-antibody formulation. European Journal of Pharmaceutics and Biopharmaceutics 59, 407-417.

Majumdar, S., Ford, B.M., Mar, K.D., Sullivan, V.J., Ulrich, R.G., D'Souza, A.J.M., 2011. Evaluation of the effect of syringe surfaces on protein formulations. Journal of Pharmaceutical Sciences 100, 2563-2573.

Morris, A.M., Watzky, M.A., Finke, R.G., 2009. Protein aggregation kinetics, mechanism, and curve-fitting: A review of the literature. Biochimica et Biophysica Acta (BBA) - Proteins \&amp; Proteomics 1794, 375-397.

Narhi, L.O., Schmit, J., Bechtold-Peters, K., Sharma, D., 2012. Classification of Protein Aggregates1. Journal of Pharmaceutical Sciences 101, 493-498.

Nashine, V.C., Kroetsch, A.M., Sahin, E., Zhou, R., Adams, M.L., 2013. Orthogonal HighThroughput Thermal Scanning Method for Rank Ordering Protein Formulations. AAPS PharmSciTech 14, 1360-1366.

Panchal, J., Kotarek, J., Marszal, E., Topp, E.M., 2014. Analyzing Subvisible Particles in Protein Drug Products: a Comparison of Dynamic Light Scattering (DLS) and Resonant Mass Measurement (RMM). The AAPS Journal 16, 440-451.

Pharmacopeia, T.U.S., 2011. The United States Pharmacopeial

Pharmacopeia, U.S., 2012a. <787> Subvisible particulate matter in therapeutic protein injections, in: Forum, P. (Ed.).

Pharmacopeia, U.S., 2012b. <788> Particulate Matter in Injections, in: Forum, P. (Ed.), p. 1930.

Philo, J.S., 2006. Is any measurement method optimal for all aggregate sizes and types? The AAPS Journal 8, E564-571.

Philo, J.S., Arakawa, T., 2009. Mechanisms of protein aggregation. Current Pharmaceutical Biotechnology 10, 348-351.

Rapra, S., 2015. The Future of Alliances and Partnerships in the Pre-filled Syringes Market to 2020. Market Research Report.

Ríos Quiroz, A., Lamerz, J., Cunha, T., Boillon, A., Adler, M., Finkler, C., Huwyler, J., Schmidt, R., Mahler, H.-C., Koulov, A.V., 2015. Factors Governing the Precision of Subvisible Particle Measurement Methods - A Case Study with a Low-Concentration Therapeutic Protein Product in a Prefilled Syringe. Pharm Res 33, 450-461.

Ripple, D.C., Dimitrova, M.N., 2012. Protein particles: What we know and what we do not know. Journal of Pharmaceutical Sciences 101, 3568-3579.

Ripple, D.C., Hu, Z., 2015. Correcting the Relative Bias of Light Obscuration and Flow Imaging Particle Counters. Pharm Res 33, 653-672.

Ripple, D.C., Narhi, L.O., 2015. Protein Particles $(0.1 \mu \mathrm{m}$ to $100 \mu \mathrm{m})$, State-of-the-Art and Emerging Technologies for Therapeutic Monoclonal Antibody Characterization Volume 2. 
Biopharmaceutical Characterization: The NISTmAb Case Study. American Chemical Society, pp. 357-386.

Rossow, M., Mantulin, W.W., Gratton, E., 2009. Spatiotemporal image correlation spectroscopy measurements of flow demonstrated in microfluidic channels. BIOMEDO 14, 024014-024014-024017.

Sharma, D., King, D., Oma, P., Merchant, C., 2010a. Micro-Flow Imaging: Flow Microscopy Applied to Sub-visible Particulate Analysis in Protein Formulations. The AAPS Journal 12, 455-464.

Sharma, D.K., King, D., Oma, P., Merchant, C., 2010b. Micro-Flow Imaging: Flow Microscopy Applied to Sub-visible Particulate Analysis in Protein Formulations. The AAPS Journal 12, 455-464.

Sharma, D.K., Oma, P., Pollo, M.J., Sukumar, M., 2010c. Quantification and characterization of subvisible proteinaceous particles in opalescent mAb Formulations Using Micro-Flow Imaging. Journal of Pharmaceutical Sciences 99, 2628-2642.

Shi, L., Ladizhansky, V., 2012. Magic angle spinning solid-state NMR experiments for structural characterization of proteins. Methods in molecular biology (Clifton, N.J.) 895, 153165.

Singh, S.K., 2013. Particulate Matter in Sterile Parenteral Products, in: Kolhe, P., Shah, M., Rathore, N. (Eds.), Sterile Product Development: Formulation, Process, Quality and Regulatory Considerations. Springer New York, New York, NY, pp. 359-409.

Singh, S.K., Afonina, N., Awwad, M., Bechtold-Peters, K., Blue, J.T., Chou, D., Cromwell, M., Krause, H.-J., Mahler, H.-C., Meyer, B.K., Narhi, L., Nesta, D.P., Spitznagel, T., 2010. An Industry Perspective on the Monitoring of Subvisible Particles as a Quality Attribute for Protein Therapeutics. Journal of Pharmaceutical Sciences 99, 3302-3321.

Strehl, R., Rombach-Riegraf, V., Diez, M., Egodage, K., Bluemel, M., Jeschke, M., Koulov, A., 2012. Discrimination Between Silicone Oil Droplets and Protein Aggregates in Biopharmaceuticals: A Novel Multiparametric Image Filter for Sub-visible Particles in Microflow Imaging Analysis. Pharm Res 29, 594-602.

Teska, B.M., Brake, J.M., Tronto, G.S., Carpenter, J.F., Aggregation and Particle Formation of Therapeutic Proteins in Contact With a Novel Fluoropolymer Surface Versus Siliconized Surfaces: Effects of Agitation in Vials and in Prefilled Syringes. Journal of Pharmaceutical Sciences.

Thirumangalathu, R., Krishnan, S., Ricci, M.S., Brems, D.N., Randolph, T.W., Carpenter, J.F., 2009. Silicone oil- and agitation-induced aggregation of a monoclonal antibody in aqueous solution. Journal of Pharmaceutical Sciences 98, 3167-3181.

TMR, 2013. Prefilled Syringes Market (Glass and Plastic) - Global Industry Analysis, Size, Volume, Share, Growth, Trends and Forecast, 2013 - 2019.

Treuheit, M., Kosky, A., Brems, D., 2002. Inverse Relationship of Protein Concentration and Aggregation. Pharm Res 19, 511-516.

V, W.L.a.V., 2011. Evaluation of end-over-end rotation/agitation of protein solutions in prefilled syringes made from glass or plastic as a preliminary indicator of protein aggregation, Protein Stability Conference, Breckenridge, Colorado, US. 
Vedadi, M., Arrowsmith, C.H., Allali-Hassani, A., Senisterra, G., Wasney, G.A., 2010. Biophysical characterization of recombinant proteins: A key to higher structural genomics success. Journal of Structural Biology 172, 107-119.

Weinbuch, D., Zoells, S., Wiggenhorn, M., Friess, W., Winter, G., Jiskoot, W., Hawe, A., 2013a. Micro-flow imaging and resonant mass measurement (archimedes) - complementary methods to quantitatively differentiate protein particles and silicone oil droplets. Journal of Pharmaceutical Sciences 102, 2152-2165.

Weinbuch, D., Zölls, S., Wiggenhorn, M., Friess, W., Winter, G., Jiskoot, W., Hawe, A., 2013b. Micro-flow imaging and resonant mass measurement (archimedes) - complementary methods to quantitatively differentiate protein particles and silicone oil droplets. Journal of Pharmaceutical Sciences 102, 2152-2165.

Wuchner, K., Büchler, J., Spycher, R., Dalmonte, P., Volkin, D.B., 2010. Development of a microflow digital imaging assay to characterize protein particulates during storage of a high concentration IgG1 monoclonal antibody formulation. Journal of Pharmaceutical Sciences 99, 3343-3361.

Zölls, S., Gregoritza, M., Tantipolphan, R., Wiggenhorn, M., Winter, G., Friess, W., Hawe, A., 2013. How subvisible particles become invisible - relevance of the refractive index for protein particle analysis. Journal of Pharmaceutical Sciences 102, 1434-1446.

Zolls, S., Tantipolphan, R., Wiggenhorn, M., Winter, G., Jiskoot, W., Friess, W., Hawe, A., 2012. Particles in therapeutic protein formulations, Part 1: Overview of analytical methods. Journal of Pharmaceutical Sciences 101, 914-935. 


\section{List of Figures}

Figure 1: a. Schematic diagram of RICS. RICS is based on the use of acquired confocal images where particles have been fluorescently labelled. Through the autocorrelation of the images, the diffusion time is determined. Depending on the timescale of the process, pixel (micro-seconds), line (milliseconds) or frame (seconds) correlation methods can be used. Adapted from (Digman et al., 2005). b. Confocal micrographs representing labelling by SYPRO® Red (Ex: $543 \mathrm{~nm}$, LP585 nm) (top) and SYPRO® Orange (Ex: $488 \mathrm{~nm}$, BP560-615nm) (bottom) to PS-20 and sloughed silicone-oil droplets from agitated syringes. Micrographs indicate no labelling to silicone-oil by SYPRO® Red and labelling of silicone-oil by SYPRO® Orange.

Figure 2: Protein particle counts in mAb PFS solutions, in the presence and absence of agitation, as a function of PS-20 concentration $(0 \%, 0.02 \%$ and $0.05 \% \mathrm{w} / \mathrm{v})$. Horizontal and vertical axis represents particle counts (particle counts per $\mathrm{mL}$ ) determined by RICS, RMM and MFI for size ranges (i) < $0.07 \mu \mathrm{m}$, (ii) $0.07-0.5 \mu \mathrm{m}$, (iii) $0.5-5 \mu \mathrm{m}$ and (iv) $>5 \mu \mathrm{m}$. Axis scale varies per technique to ease visualisation of data. Values represent average counts and error bars represent the std. dev. for $n=3$.

Figure 3: Silicone-oil droplet counts in buffer-filled PFS solutions, in the presence and absence of agitation, as a function of PS-20 concentration $(0 \%, 0.02 \%$ and $0.05 \% \mathrm{w} / \mathrm{v})$. Horizontal and vertical axis represents particle counts (particle counts per $\mathrm{mL}$ ) determined by RICS, RMM and MFI for size ranges (i) $<0.07 \mu \mathrm{m}$, (ii) $0.07-0.5 \mu \mathrm{m}$, (iii) $0.5-5 \mu \mathrm{m}$ and (iv) $>5 \mu \mathrm{m}$. Axis scale varies per technique to ease visualisation of data. Values represent average counts and error bars represent the std. dev. for $\mathrm{n}=3$.

Figure 4: Silicone-oil droplet counts in mAb PFS solutions, in the presence and absence of agitation, as a function of PS-20 concentration $(0 \%, 0.02 \%$ and $0.05 \% \mathrm{w} / \mathrm{v})$. Horizontal and vertical axis represents particle counts (particle counts per $\mathrm{mL}$ ) determined by RMM and MFI for size ranges (i) < $0.07 \mu \mathrm{m}$, (ii) $0.07-0.5 \mu \mathrm{m}$, (iii) $0.5-5 \mu \mathrm{m}$ and (iv) $>5 \mu \mathrm{m}$. Axis scale varies per technique to ease visualisation of data. Values represent average counts and error bars represent the std. dev. for $n=3$. 

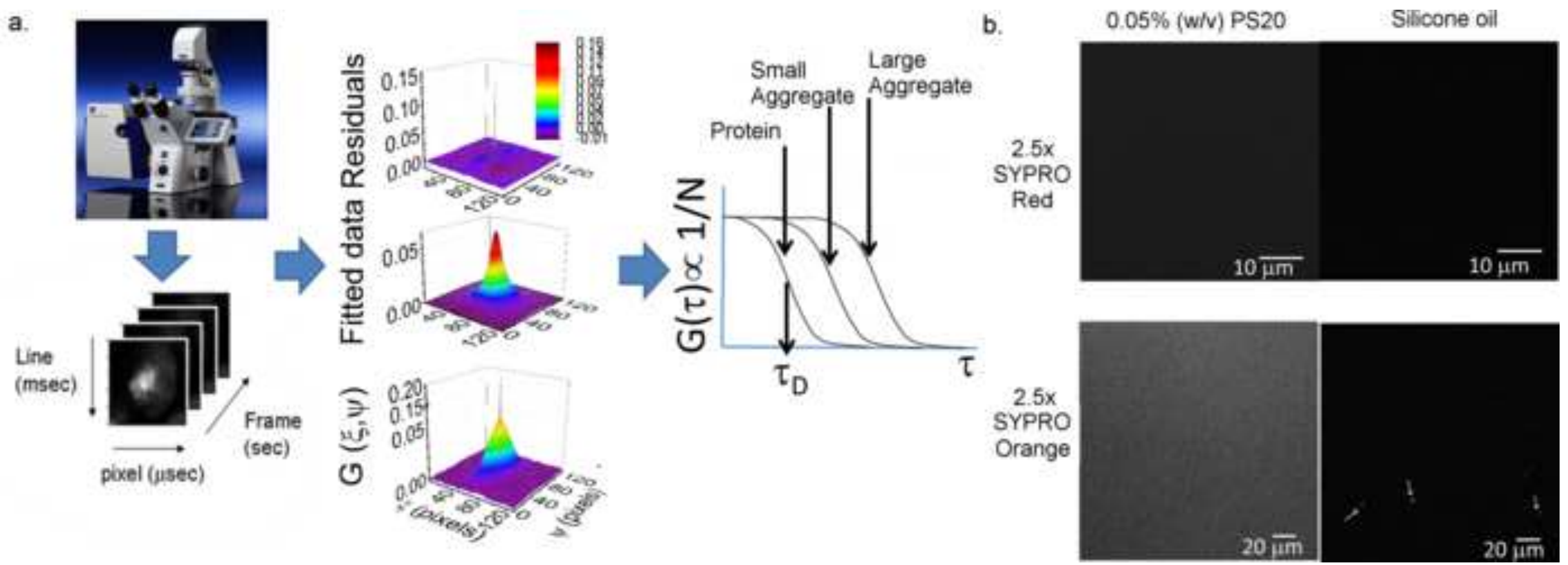


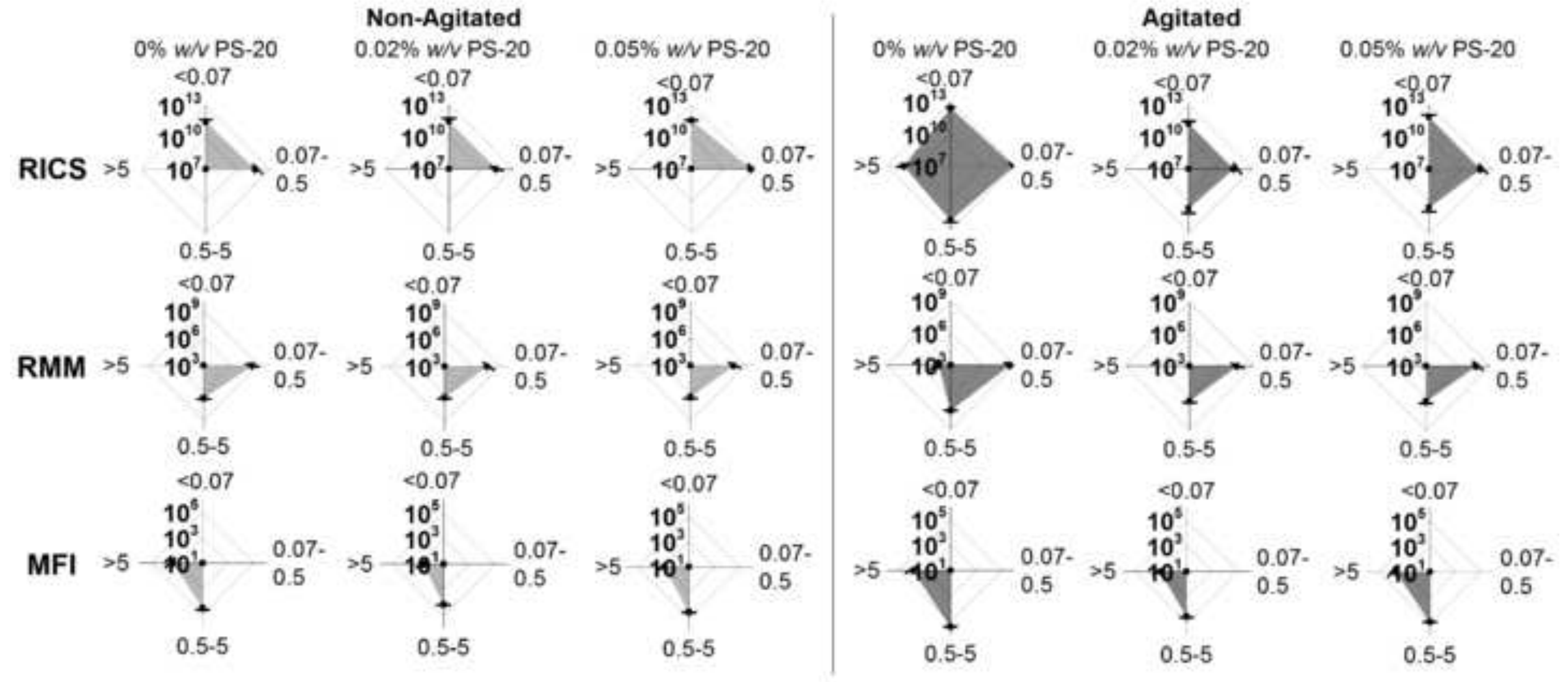



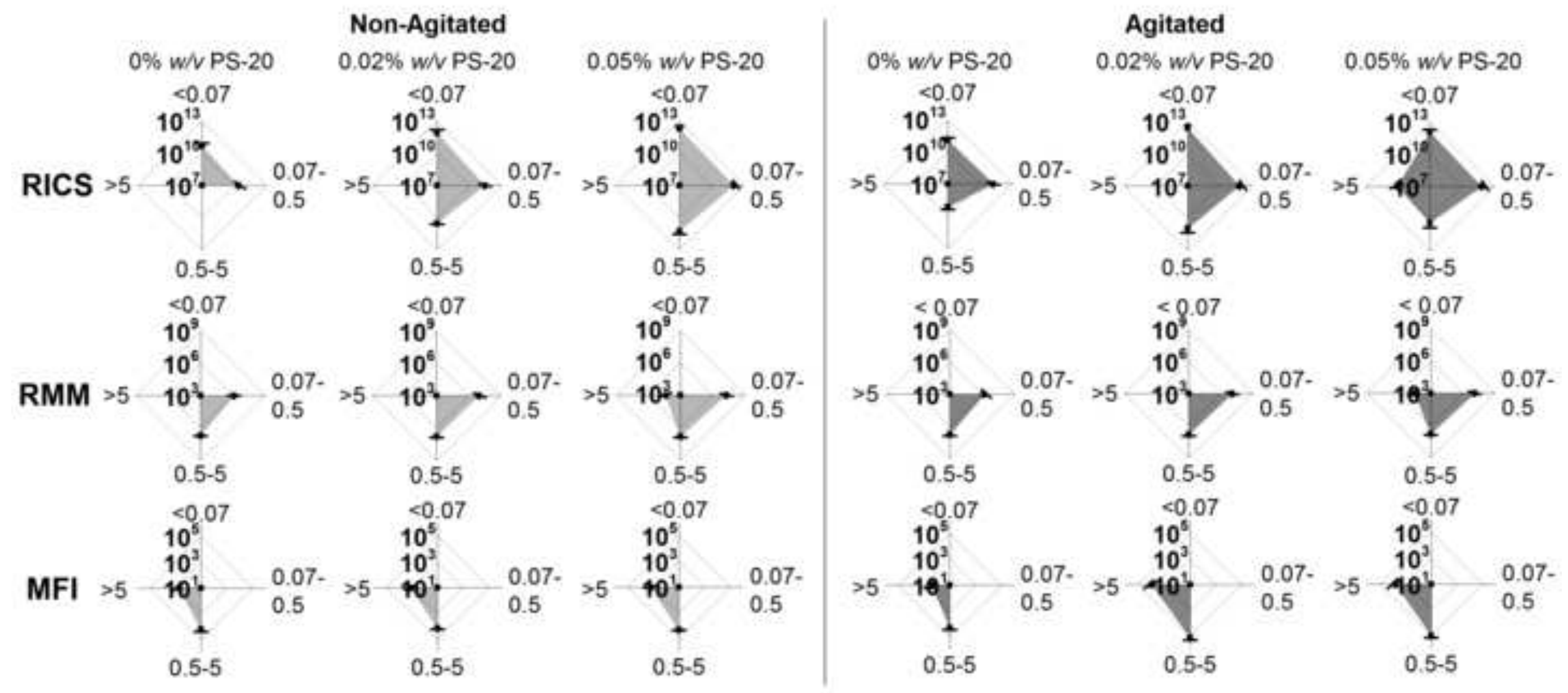


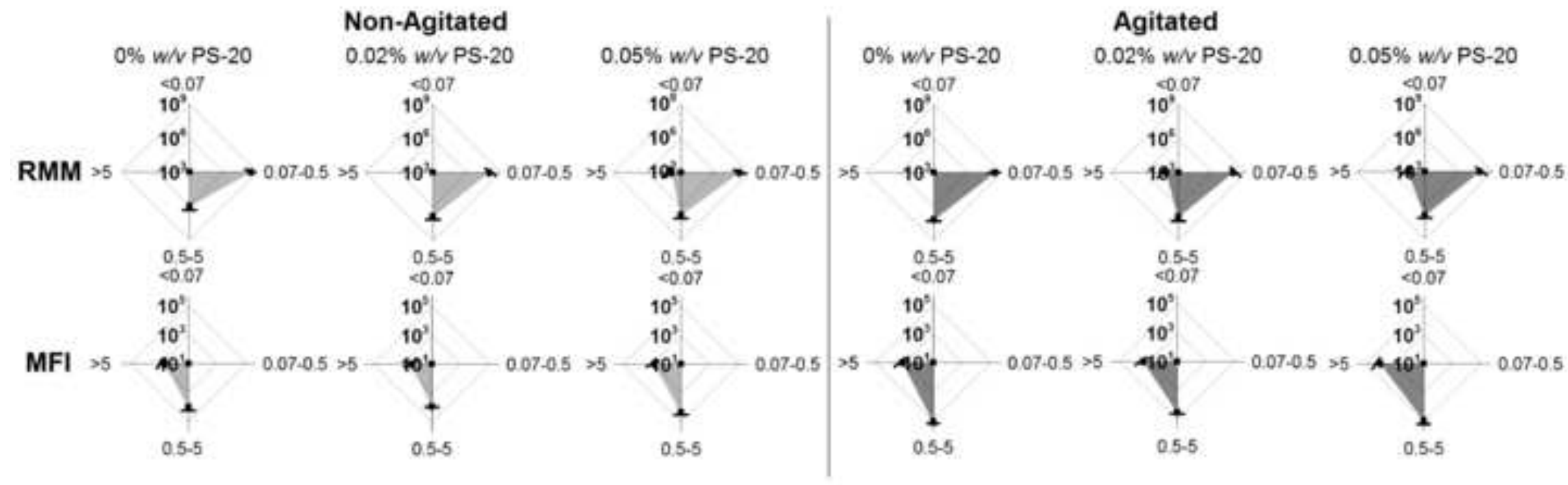



Supplementary Material
Click here to download S Supplementary Material
Click here to download Supplementary Material: Shah Paper SUPPLEMENTARY INFORMATION FINAL VERSION JAN_2016.docx 


\section{IJP AUTHOR CHECKLIST}

Dear Author,

It frequently happens that on receipt of an article for publication, we find that certain elements of the manuscript, or related information, is missing. This is regrettable of course since it means there will be a delay in processing the article while we obtain the missing details.

In order to avoid such delays in the publication of your article, if accepted, could you please run through the list of items below and make sure you have completed the items.

\section{Overall Manuscript Details}

- Is this the final revised version?

- Are all text pages present?

- Are the corresponding author's postal address, telephone and fax numbers complete on the manuscript?

- Have you provided the corresponding author's e-mail address?

- Manuscript type - please check one of the following:

Full-length article

Review article

Rapid Communication

Note

Letter to the Editor

Other

- Manuscript section - paper to be published in:

Pharmaceutical Nanotechnology section

Personalised Medicine section

\section{Manuscript elements}

- Short summary/abstract enclosed?

- 3-6 Keywords enclosed?

- Complete reference list enclosed?

- Is the reference list in the correct journal style?

- Are all references cited in the text present in the reference list?

- Are all original figures cited in the text enclosed?

Electronic artwork format?

- Are figure legends supplied?

- Are all figures numbered and orientation provided?

- Are any figures to be printed in colour?

If yes, please list which figures here:

- If applicable, are you prepared to pay for reproduction in colour?

- Are all tables cited in the text supplied?

\section{General}

- Can you accept pdf proofs sent via e-mail? 